Discrete Comput Geom 28:211-222 (2002)

DOI: $10.1007 / \mathrm{s} 00454-002-2808-2$

\title{
Perfect Partitions of Convex Sets in the Plane
}

\author{
Atsushi Kaneko ${ }^{1}$ and M. Kano ${ }^{2}$ \\ ${ }^{1}$ Department of Computer Science and Communication Engineering, \\ Kogakuin University, Nishi-Shinjuku, Shinjuku-ku, \\ Tokyo 163-8677, Japan \\ kaneko@ee.kogakuin.ac.jp \\ ${ }^{2}$ Department of Computer and Information Sciences, Ibaraki University, \\ Hitachi, Ibaraki 316-8511, Japan \\ kano@cis.ibaraki.ac.jp
}

\begin{abstract}
For a region $X$ in the plane, we denote by area $(X)$ the area of $X$ and by $\ell(\partial(X))$ the length of the boundary of $X$. Let $S$ be a convex set in the plane, let $n \geq 2$ be an integer, and let $\alpha_{1}, \alpha_{2}, \ldots, \alpha_{n}$ be positive real numbers such that $\alpha_{1}+\alpha_{2}+\cdots+\alpha_{n}=1$ and $0<\alpha_{i} \leq \frac{1}{2}$ for all $1 \leq i \leq n$. Then we shall show that $S$ can be partitioned into $n$ disjoint convex subsets $T_{1}, T_{2}, \ldots, T_{n}$ so that each $T_{i}$ satisfies the following three conditions: (i) $\operatorname{area}\left(T_{i}\right)=\alpha_{i} \times \operatorname{area}(S)$; (ii) $\ell\left(T_{i} \cap \partial(S)\right)=\alpha_{i} \times \ell(\partial(S))$; and (iii) $T_{i} \cap \partial(S)$ consists of exactly one continuous curve.
\end{abstract}

\section{Introduction}

We begin with a motivation of the original problem related to our results. Some children attend a birthday party, and there is a big non-circular birthday cake. We want to divide the cake among all the children in such a way that each child gets the same amount of cake and the same amount of icing (exposed area) and holds it easily (i.e., each cake is convex and has exactly one icing side) [1]. If the height of the cake is constant, then the above problem can be said as follows. Let $S$ be a convex set in the plane, which corresponds to the base of the cake. Then is it possible to partition $S$ into $n$ convex subsets so that each subset has the same area and has exactly one continuous part of the boundary of $S$ with the same length (Fig. 1)? If such a partition exists, we say that $S$ can be perfectly partitioned into $n$ convex subsets, and call this partition a perfect $n$-partition.

It was proved in [2] that a perfect partition always exists for every $n \geq 3$, that is, the following theorem was obtained. 


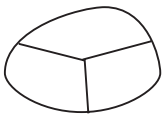

(a)

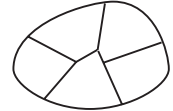

(b)

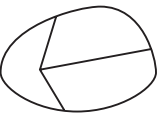

(c)

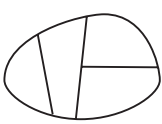

(d)

Fig. 1. (a), (b) Perfect partitions and (c), (d) non-perfect partitions.

Theorem 1 [2]. Every convex set in the plane has a perfect n-partition for every integer $n \geq 3$ (Fig. 1).

For a domain $X$ in the plane, we denote by $\operatorname{area}(X)$ the area of $X$ and by $\partial(X)$ the boundary of $X$. For a curve $C$ in the plane, $\ell(C)$ denotes the length of $C$. In particular, $\ell(\partial(X))$ denotes the length of the boundary of $X$.

In this paper we prove the following Theorem 2, which is a generalization of Theorem 1, and the partition given in Theorem 2 is called a generalized perfect n-partition.

Theorem 2. Let $S$ be a convex set in the plane, let $n \geq 2$ be an integer, and let $\alpha_{1}, \alpha_{2}, \ldots, \alpha_{n}$ be positive real numbers such that $\alpha_{1}+\alpha_{2}+\cdots+\alpha_{n}=1$ and $0<\alpha_{i} \leq \frac{1}{2}$ for all $1 \leq i \leq n$. Then $S$ can be partitioned into $n$ convex subsets $T_{1}, T_{2}, \ldots, T_{n}$ so that each $T_{i}$ satisfies the following three conditions: (i) $\operatorname{area}\left(T_{i}\right)=\alpha_{i} \times \operatorname{area}(S)$; (ii) $\ell\left(T_{i} \cap \partial(S)\right)=\alpha_{i} \times \ell(\partial(S))$; and (iii) $T_{i} \cap \partial(S)$ consists of exactly one continuous curve (Fig. 2).

If $\frac{1}{2}<\alpha_{1}<1$ and $\alpha_{1}+\alpha_{2}=1$, then it is impossible to partition a circle $C$ into two subsets satisfying the conditions of Theorem 2 since the area of a convex subset $T_{1}$ with $\ell\left(T_{1} \cap \partial(C)\right)=\alpha_{1} \times \ell(\partial(C))$ is always greater than $\alpha_{1} \times$ area $(C)$. Hence we need the condition that $\alpha_{i} \leq \frac{1}{2}$ for all $i$.

We now explain the relationship between a perfect $n$-partition and the result on balanced partitions of two sets of points in the plane. The following theorem was conjectured and proved for $n=1,2$ in [6] and [7], and was recently proved independently for every $n \geq 1$ in [4], [5], and [9]. Note that other interesting results related to our topic are found in [3], where partitions by fans are considered.

Theorem 3 [4], [5], [9]. Let $m \geq 1, n \geq 1$ and $k \geq 2$ be positive integers. Let $R$ be a set of $m k$ red points and $B$ a set of $n k$ blue points in the plane such that no three points

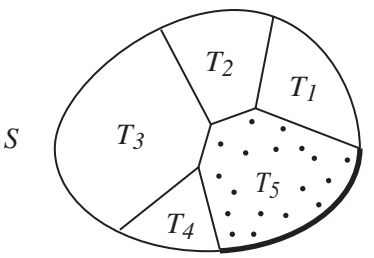

Fig. 2. A generalized perfect 5-partition of a convex set $S$ with the emphasis of $T_{5}$ and $T_{5} \cap \partial(S)$. 
of $R \cup B$ lie on the same line. Then $R \cup B$ can be partitioned into $k$ disjoint subsets $X_{1}, X_{2}, \ldots, X_{k}$ so that every $X_{i}(1 \leq i \leq k)$ contains exactly $m$ red points and $n$ blue points, and $\operatorname{conv}\left(X_{i}\right) \cap \operatorname{conv}\left(X_{j}\right)=\emptyset$ for all $i \neq j$, where $\operatorname{conv}\left(X_{i}\right)$ denotes the convex hull of $X_{i}$.

For a given convex set $S$ in the plane, if we uniformly put a lot of red points on $\partial(S)$ and a lot of blue points on $S$, then by the above Theorem 3, we can partition $S$ into $k$ convex subsets $\left\{X_{i}\right\}$ so that each $X_{i}$ contains the same number of red points and the same number of blue points, that is, the length of $X_{i} \cap \partial(S)$ is constant and the area of $X_{i}$ is also constant. However, we cannot say that $X_{i} \cap \partial(S)$ consists of exactly one continuous curve (Fig. 1(d)). Thus even a perfect $n$-partition cannot be obtained directly from Theorem 3.

We conclude this section with a remark on Theorem 4 and a conjecture. When we consider a convex polygon in the plane instead of a convex set, we can similarly partition the convex polygon into some convex polygons under weaker conditions. This partition is given in Theorem 4 . The following conjecture is a generalization of Theorem 3. Note that it is shown in [8] that if either $m_{1}+m_{2}+\cdots+m_{k} \leq 8$, or $1 \leq m_{i} \leq 2$ for every $1 \leq i \leq k$, then the conjecture holds.

Conjecture A. Let $k \geq 1$ be a positive integer. Let $m_{1} \geq m_{2} \geq \cdots \geq m_{k} \geq 1$ and $n_{1} \geq n_{2} \geq \cdots \geq n_{k} \geq 1$ be positive integers such that $m_{1} \leq\left(m_{1}+m_{2}+\cdots+m_{k}\right) / 3$ and

$$
\frac{n_{1}}{m_{1}}=\frac{n_{2}}{m_{2}}=\cdots=\frac{n_{k}}{m_{k}} .
$$

Let $R$ be a set of $m_{1}+m_{2}+\cdots+m_{k}$ red points and let $B$ be a set of $n_{1}+n_{2}+\cdots+n_{k}$ blue points in the plane such that no three points of $R \cup B$ lie on the same line. Then $R \cup B$ can be partitioned into $k$ disjoint subsets $X_{1}, X_{2}, \ldots, X_{k}$ so that each $X_{i}$ contains exactly $m_{i}$ red points and $n_{i}$ blue points and $\operatorname{conv}\left(X_{i}\right) \cap \operatorname{conv}\left(X_{j}\right)=\emptyset$ for all $i \neq j$.

\section{Proof of Theorem 2}

We define some notations. For two points $X$ and $Y$ in the plane, we denote by $X Y$ the straight-line segment joining $X$ to $Y$ and by $|X Y|$ the length of $X Y$, which is equal to the distance between $X$ and $Y$.

A quadrilateral with consecutive vertices $\left(P_{1}, P_{2}, P_{3}, P_{4}\right)$, a hexagon with consecutive vertices $\left(Q_{1}, Q_{2}, \ldots, Q_{6}\right)$, and an octagon with consecutive vertices $\left(R_{1}, R_{2}, \ldots, R_{8}\right)$ are denoted by quad $\left(P_{1} P_{2} P_{3} P_{4}\right)$, hex $\left(Q_{1} Q_{2} \cdots Q_{6}\right)$, and octagon $\left(R_{1} R_{2} \cdots R_{8}\right)$, respectively.

We begin with a theorem on partitions of convex polygons, which might be of interest in itself and is used in the proof of Theorem 2.

Theorem 4. Let $n$ and $m$ be integers such that $3 \leq n$ and $1 \leq m \leq n$. Let $P$ be a convex polygon in the plane with $n$ vertices, and let $\beta_{1}, \beta_{2}, \ldots, \beta_{m}$ be positive real numbers such that $\beta_{1}+\beta_{2}+\cdots+\beta_{m}=\operatorname{area}(P)$. Then for given $m$ edges $e_{1}, e_{2}, \ldots, e_{m}$ 

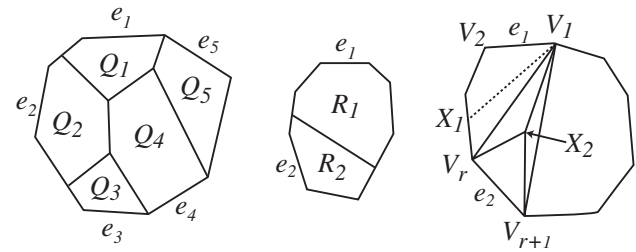

Fig. 3. A partition $\left\{Q_{i}\right\}$ of $P$, and the figure in the proof.

of $P, P$ can be partitioned into $m$ disjoint convex polygons $Q_{1}, Q_{2}, \ldots, Q_{m}$ so that each $Q_{i}(1 \leq i \leq m)$ contains the edge $e_{i}$ and has area $\beta_{i}$ (Fig. 3).

Proof. We prove the theorem by induction on $m$. If $m=1$, then $Q_{1}=P$ is the desired partition. If $m=2$, then there exists a line that partition $P$ into two sub-polygons $R_{1}$ and $R_{2}$ in such a way that $R_{1}$ contains $e_{1}$ but not $e_{2}$ and has area $\beta_{1}$, which gives us the desired partition. So we may assume that $m \geq 3$.

Let $V_{1}, V_{2}, \ldots, V_{n}$ be the consecutive vertices of $P$. By a new labeling of $\left\{V_{i}\right\}$ and $\left\{\beta_{i}\right\}$, we may assume that $e_{1}=V_{1} V_{2}, e_{2}=V_{r} V_{r+1}$ and no edges of $P$ between $V_{2}$ and $V_{r}$ are chosen in $\left\{e_{i}\right\}$.

Let $P_{1}$ be the sub-polygon with vertex set $\left\{V_{1}, V_{2}, \ldots, V_{r}\right\}$, which is obtained from $P$ by dividing by the diagonal $V_{1} V_{r}$ (Fig. 3). If area $\left(P_{1}\right) \geq \beta_{1}$, then we can find a point $X_{1}$ on the edges $V_{2} V_{3} \cup \cdots \cup V_{r-1} V_{r}$ such that the area of the sub-polygon divided by $V_{1} X_{1}$ is equal to $\beta_{1}$. Then we can apply the inductive hypothesis to the remaining polygon. Therefore we may assume that $\operatorname{area}\left(P_{1}\right)<\beta_{1}$.

Let $P_{2}$ be the sub-polygon with vertex set $\left\{V_{1}, V_{2}, \ldots, V_{r}, V_{r+1}\right\}$. If $\operatorname{area}\left(P_{2}\right) \geq$ $\beta_{1}+\beta_{2}$, then area $\left(\triangle V_{1} V_{r} V_{r+1}\right) \geq \beta_{1}-\operatorname{area}\left(P_{1}\right)+\beta_{2}$, and so we can easily find a point $X_{2}$ in $\triangle V_{1} V_{r} V_{r+1}$ such that

$$
\operatorname{area}\left(\triangle X_{2} V_{1} V_{r}\right)=\beta_{1}-\operatorname{area}\left(P_{1}\right) \text { and } \operatorname{area}\left(\triangle X_{2} V_{r} V_{r+1}\right)=\beta_{2} \text {, }
$$

which implies that the convex polygon $P_{1}+\triangle X_{2} V_{1} V_{r}$ has area $\beta_{1}$ (Fig. 3). Then we apply the inductive hypothesis to the remaining convex polygon, and get the desired partition of $P$. Hence we may assume that area $\left(P_{2}\right)<\beta_{1}+\beta_{2}$.

Put $\gamma=\beta_{1}+\beta_{2}-$ area $\left(P_{2}\right)>0$. We consider the polygon $P-P_{2}$ together with the edges $\left\{V_{1} V_{r+1}, e_{3}, \ldots, e_{m}\right\}$ and the positive real numbers $\gamma, \beta_{3}, \ldots, \beta_{m}$. Then by the inductive hypothesis, $P-P_{2}$ can be partitioned into $m-1$ convex subsets $R, Q_{3}, \ldots, Q_{m}$. It is easy to see that $R \cup P_{2}$ is a convex polygon with area $\beta_{1}+\beta_{2}$, and can be partitioned into two convex polygons that contain $e_{1}$ and $e_{2}$, respectively, and have areas $\beta_{1}$ and $\beta_{2}$, respectively. Consequently, the theorem is proved.

In order to prove our theorem, we need some lemmas. The following lemma was proved in [2].

Lemma 5. Let $\triangle A B C$ be a triangle in the plane, and let $S$ be a convex set that is contained in $\triangle A B C$ and contains $B C$. Let $\operatorname{arc}(B C)=\partial(S)-B C$. If $\angle B \geq \angle C$, then 

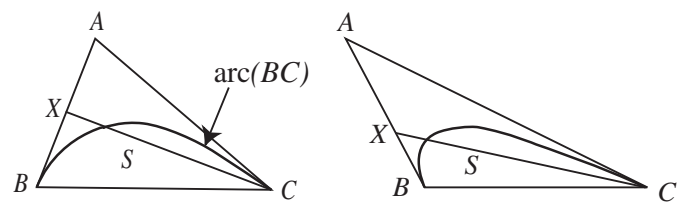

Fig. 4. Triangles $\triangle A B C$ and convex sets $S$.

for a point $X$ on $A B$ such that $|B X|+|X C|=\ell(\operatorname{arc}(B C))$, it follows that area $(\triangle X B C) \leq$ area(S) (Fig. 4).

Lemma 6. Let $\triangle A B C, S$ and $\operatorname{arc}(B C)$ be the same as Lemma 5 above. Let $h$ denote the height of $\triangle A B C$ relative to base $A B$ or $A C$ (Fig. 5(a)-(c)). Then

$$
\operatorname{area}(S)<\frac{1}{2} h \times \ell(\operatorname{arc}(B C)) .
$$

Proof. Without loss generality, we may assume that $h$ is the height of $\triangle A B C$ relative to base $A B$. Let $D$ be the foot of the perpendicular dropped from $C$ to the line containing $A B$. Then $h=|C D|$. We first assume that $\angle B \leq \pi / 2$, that is, $\angle B$ is acute (Fig. 5(a),(b)).

If $D$ is outside of $\triangle A B C$, then

$$
\operatorname{area}(S) \leq \operatorname{area}(\triangle A B C)=\frac{1}{2}|A B| h<\frac{1}{2} h \times \ell(\operatorname{arc}(B C)) .
$$

Thus we may assume that $D$ lies on $A B$. Let $E$, if any, be the intersection of $C D$ and $\operatorname{arc}(B C)$ (Fig. 5(d)). If $E$ does not exist, then $S$ is contained in $\triangle D B C$, and so area $(S) \leq$ $\operatorname{area}(\triangle D B C)=(h / 2)|B D|<(h / 2) \times \ell(\operatorname{arc}(B C))$. Thus we may assume that the intersection $E$ exists. Then $S$ is divided into two subset $S_{1}=S \cap \triangle A D C$ and $S_{2}=$ $S \cap \triangle D B C$ by the line $C D$. We have

$$
\operatorname{area}\left(S_{2}\right) \leq \triangle D B C=\frac{1}{2} h|D B| \leq \frac{1}{2} h \times \ell(\operatorname{arc}(B E)) .
$$

Since $S_{1}$ is clearly contained in a rectangle $R$ with edge $C D$ and height $\ell(\operatorname{arc}(C E)) / 2$, it follows that

$$
\operatorname{area}\left(S_{1}\right)<\operatorname{area}(R)=\frac{1}{2} h \times \ell(\operatorname{arc}(E C)) .
$$

Therefore we get the desired inequality in this case.

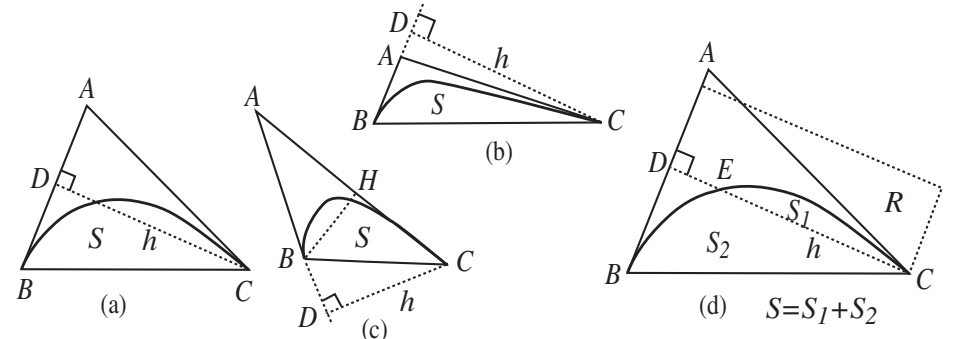

Fig. 5. Triangles $\triangle A B C$ and convex sets $S$. 

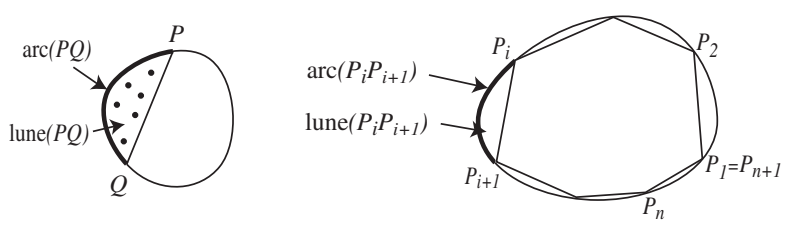

Fig. 6. $\operatorname{Arc}(P Q)$, lune $(P Q), \operatorname{arc}\left(P_{i} P_{i+1}\right)$ and lune $\left(P_{i} P_{i+1}\right)$.

Next suppose that $\angle B>\pi / 2$ (Fig. 5(c)). Let $H$ be the foot of the perpendicular dropped from $B$ to line $A C$. In this case we can show that the following inequality holds by the same argument as above:

$$
\operatorname{area}(S)<\frac{1}{2}|B H| \times \ell(\operatorname{arc}(B C)) .
$$

Since $h=|C D|>|B H|$, the above inequality implies the desired inequality (1) of the lemma.

For two points $P$ and $Q$ on the boundary of a convex set $S$, the boundary of $S$ is divided into two arcs by $P$ and $Q$, and $\operatorname{arc}(P Q)$ denotes one of the $\operatorname{arcs}$ between $P$ and $Q$ that is easily determined from the context and is the shorter one in almost every case (Fig. 6). If it is not easily determined, we explain it more precisely. Moreover, we denote by lune $(P Q)$ the lune surrounded by the $\operatorname{arc} \operatorname{arc}(P Q)$ and by the line segment $P Q$ (Fig. 6).

Lemma 7. Let $n \geq 3$ be an integer, and let $S$ and $\alpha_{1}, \alpha_{2}, \ldots, \alpha_{n}$ be the same as in Theorem 2. Let $P_{1}, P_{2}, \ldots, P_{n}$ be $n$ points on $\partial(S)$ such that for every $1 \leq i \leq n$, $\ell\left(\operatorname{arc}\left(P_{i} P_{i+1}\right)\right)=\alpha_{i} \times \ell(\partial(S))$. Then area(lune $\left.\left(P_{i} P_{i+1}\right)\right)<\alpha_{i} \times$ area $(S)$ for all $1 \leq$ $i \leq n$ except at most one certain integer, where $P_{n+1}=P_{1}$ (Figure 6 ).

Proof. Suppose that the lemma does not hold. By a new suitable labeling of $\left\{P_{i}\right\}$, we may assume that there exist $n$ points $P_{1}, P_{2}, \ldots, P_{n}$ on $\partial(S)$ such that $\ell\left(\operatorname{arc}\left(P_{i} P_{i+1}\right)\right)=$ $\alpha_{i} \times \ell(\partial(S))$ for all $1 \leq i \leq n$,

$$
\operatorname{area}\left(\text { lune }\left(P_{1} P_{2}\right)\right) \geq \alpha_{1} \operatorname{area}(S) \text { and } \operatorname{area}\left(\operatorname{lune}\left(P_{r} P_{r+1}\right)\right) \geq \alpha_{r} \operatorname{area}(S)
$$

for some $r, 2 \leq r \leq n$. We first consider the case that $3 \leq r \leq n-2$ (i.e., the case where $\operatorname{arc}\left(P_{1} P_{2}\right)$ and $\operatorname{arc}\left(P_{r} P_{r+1}\right)$ have no common vertex).

Since $S$ is a convex set, there exist lines tangent to $S$ at $P_{1}, P_{2}, P_{r}$ and $P_{r+1}$, respectively. We first consider the case where these four lines makes a quadrilateral, that is, we first assume that the quadrilateral quad $\left(B_{1} B_{2} B_{3} B_{4}\right)$ given in Fig. 7 exists.

Consider the triangle $\triangle P_{1} P_{r+1} B_{1}$ and the convex subset $S \cap \triangle P_{1} P_{r+1} B_{1}$. Without loss of generality, we may assume that $\angle P_{1} \leq \angle P_{r+1}$ since otherwise we can apply the same argument to $B_{1} P_{1}$ instead of $B_{1} P_{r+1}$. Let $Y$ be the point on $B_{1} P_{r+1}$ such that $\left|P_{1} Y\right|+\left|Y P_{r+1}\right|=\ell\left(\operatorname{arc}\left(P_{1} P_{r+1}\right)\right)$. Then by Lemma 5, we have

$$
\operatorname{area}\left(\triangle Y P_{1} P_{r+1}\right) \leq \operatorname{area}\left(S \cap \triangle P_{1} P_{r+1} B_{1}\right) .
$$



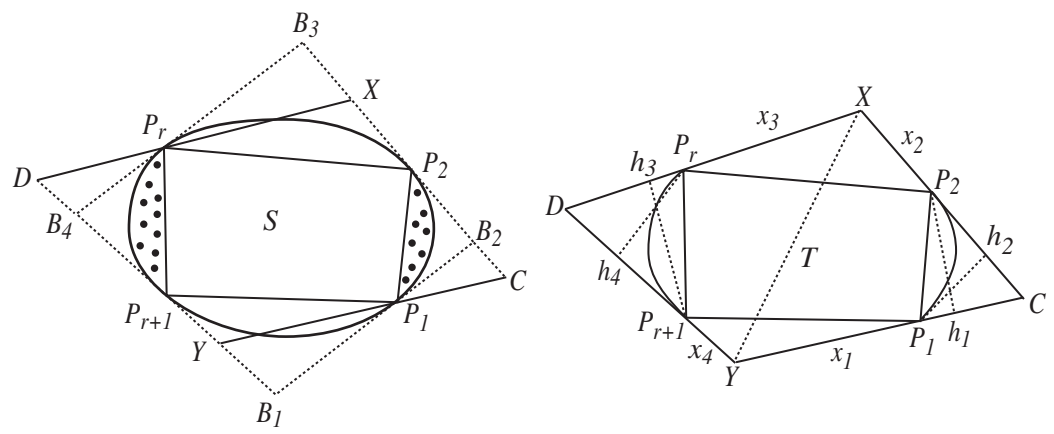

Fig. 7. Quad $\left(B_{1} B_{2} B_{3} B_{4}\right)$ and the convex set $T$.

By the same argument as above, for the convex subset $S \cap \triangle P_{2} B_{3} P_{r}$ and for the point $X$ on $P_{2} B_{3}\left(\right.$ or $\left.B_{3} P_{r}\right)$ with $\left|P_{2} X\right|+\left|X P_{r}\right|=\ell\left(\operatorname{arc}\left(P_{2} P_{r}\right)\right)$, we have

$$
\text { area } \triangle X P_{r} P_{2} \leq \operatorname{area}\left(S \cap \triangle P_{2} B_{3} P_{r}\right) .
$$

Let

$$
T:=\left(S-\left(\text { lune }\left(P_{2} P_{r}\right) \cup \operatorname{lune}\left(P_{r+1} P_{1}\right)\right)\right) \cup \triangle Y P_{1} P_{r+1} \cup \triangle X P_{r} P_{2}
$$

(Fig. 7). Then $T$ is a convex set with $\ell(\partial(T))=\ell(\partial(S))$ and $\operatorname{area}(T) \leq \operatorname{area}(S)$, and is contained in a quadrilateral quad $(C X D Y)$. The following equalities and inequalities immediately hold:

$$
\begin{aligned}
& \ell\left(\operatorname{arc}\left(P_{1} P_{2}\right)\right)=\alpha_{1} \ell(\partial(T)), \quad \ell\left(\operatorname{arc}\left(P_{r} P_{r+1}\right)\right)=\alpha_{r} \ell(\partial(T)), \\
& \text { area }\left(\text { lune }\left(P_{1} P_{2}\right)\right) \geq \alpha_{1} \operatorname{area}(T), \quad \operatorname{area}\left(\operatorname{lune}\left(P_{r} P_{r+1}\right)\right) \geq \alpha_{r} \operatorname{area}(T) \text {. }
\end{aligned}
$$

Put $\ell^{*}=\ell(\partial(T)), x_{1}=\left|Y P_{1}\right|, x_{2}=\left|X P_{2}\right|, x_{3}=\left|X P_{r}\right|, x_{4}=\left|Y P_{r+1}\right|$ and $a=$ $\ell\left(\operatorname{arc}\left(P_{1} P_{2}\right)\right)=\alpha_{1} \ell^{*}, b=\ell\left(\operatorname{arc}\left(P_{r} P_{r+1}\right)\right)=\alpha_{r} \ell^{*}$. Let $h_{1}$ and $h_{2}$ the heights of $\triangle C P_{2} P_{1}$ relative to bases $C P_{1}$ and $C P_{2}$, respectively, and let $h_{3}$ and $h_{4}$ be the heights of $\triangle D P_{r+1} P_{r}$ relative to bases $D P_{r}$ and $D P_{r+1}$, respectively. Then we obtain the following inequalities by Lemma 6:

$$
\begin{aligned}
\operatorname{area}\left(\text { lune }\left(P_{1} P_{2}\right)\right)<\frac{1}{2} a h_{1}, & \operatorname{area}\left(\operatorname{lune}\left(P_{1} P_{2}\right)\right)<\frac{1}{2} a h_{2}, \\
\operatorname{area}\left(\text { lune }\left(P_{r} P_{r+1}\right)\right)<\frac{1}{2} & b h_{3}, \quad \operatorname{area}\left(\text { lune }\left(P_{r} P_{r+1}\right)\right)<\frac{1}{2} b h_{4}, \\
\operatorname{area}\left(\operatorname{quad}\left(X Y P_{1} P_{2}\right)\right) & =\operatorname{area}\left(\triangle X P_{1} P_{2}\right)+\operatorname{area}\left(\triangle X Y P_{1}\right) \\
& \geq \operatorname{area}\left(\triangle X P_{1} P_{2}\right)+\operatorname{area}\left(\triangle Y P_{1} P_{2}\right) \\
& =\frac{1}{2}\left(x_{2} h_{2}+x_{1} h_{1}\right), \\
\operatorname{area}\left(q u a d\left(X P_{r} P_{r+1} Y\right)\right) & \geq \operatorname{area}\left(\triangle X P_{r} P_{r+1}\right)+\operatorname{area}\left(\triangle Y P_{r} P_{r+1}\right) \\
& =\frac{1}{2}\left(x_{3} h_{3}+x_{4} h_{4}\right) .
\end{aligned}
$$


By symmetry, we may assume that $h_{1}$ is the smallest among all the $h_{i}$ 's. Then

$$
\begin{aligned}
& \operatorname{area}\left(\operatorname{hex}\left(X P_{r} P_{r+1} Y P_{1} P_{2}\right)\right) \geq \frac{1}{2}\left(x_{1}+x_{2}+x_{3}+x_{4}\right) h_{1} \\
&=\frac{1}{2}\left(1-\alpha_{1}-\alpha_{r}\right) \ell^{*} h_{1}, \\
& \operatorname{area}\left(\operatorname{hex}\left(X P_{r} P_{r+1} Y P_{1} P_{2}\right)\right)=\operatorname{area}(T)-\operatorname{area}\left(\operatorname{lune}\left(P_{1} P_{2}\right)\right)-\operatorname{area}\left(\operatorname{lune}\left(P_{r} P_{r+1}\right)\right) \\
& \leq\left(1-\alpha_{1}-\alpha_{r}\right) \operatorname{area}(T) \leq\left(1-\alpha_{1}-\alpha_{r}\right) \operatorname{area}(S) .
\end{aligned}
$$

Hence $\ell^{*} h_{1} / 2 \leq \operatorname{area}(S)$, and thus

$$
\operatorname{area}\left(\operatorname{lune}\left(P_{1} P_{2}\right)\right) \geq \alpha_{1} \operatorname{area}(S) \geq \frac{\alpha_{1} \ell^{*} h_{1}}{2} .
$$

However, this contradicts the fact that

$$
\operatorname{area}\left(\operatorname{lune}\left(P_{1} P_{2}\right)\right)<\frac{1}{2} a h_{1}=\frac{\alpha_{1} \ell^{*} h_{1}}{2} \quad(\text { by (2)). }
$$

We next assume that the quadrilateral quad $\left(B_{1} B_{2} B_{3} B_{4}\right)$ does not exist, that is, we assume that the configuration given in Figs. 8 or 9 occurs. We first consider the case of Fig. 8. Let $B_{1}, B_{2}, B_{3}$ be the intersections of lines tangent to $S$ at $P_{2}, P_{r}, P_{r+1}, P_{1}$. We take two points $X$ and $Y$ on $P_{2} B_{1} \cup B_{1} P_{r}$ and $P_{r+1} B_{3} \cup B_{3} P_{1}$, respectively, which satisfy the conditions of Lemma 5. Let $D$ be the intersection of the two lines containing $X P_{r}$ and $Y P_{r+1}$, respectively. Let $h_{1}$ and $h_{2}$ be the heights of $\triangle P_{r} D P_{r+1}$ relative to bases $D P_{r+1}$ and $D P_{r}$, respectively. Then by Lemmas 5 and 6 , we have

$$
\begin{aligned}
& \operatorname{area}\left(\operatorname{lune}\left(P_{r} P_{r+1}\right)\right)<\frac{1}{2} h_{1} \ell\left(\operatorname{arc}\left(P_{r} P_{r+1}\right)\right), \\
& \operatorname{area}\left(\operatorname{lune}\left(P_{r} P_{r+1}\right)\right)<\frac{1}{2} h_{2} \ell\left(\operatorname{arc}\left(P_{r} P_{r+1}\right)\right), \\
&\left|P_{2} X\right|+\left|X P_{r}\right|=\ell\left(\operatorname{arc}\left(P_{2} P_{r}\right)\right), \operatorname{area}\left(\triangle P_{2} X P_{r}\right) \leq \operatorname{area}\left(\operatorname{lune}\left(P_{2} P_{r}\right)\right), \\
&\left|P_{r+1} Y\right|+\left|Y P_{1}\right|=\ell\left(\operatorname{arc}\left(P_{r+1} P_{1}\right)\right), \quad \operatorname{area}\left(\triangle P_{r+1} Y P_{1}\right) \leq \operatorname{area}\left(\operatorname{lune}\left(P_{r+1} P_{1}\right)\right) .
\end{aligned}
$$
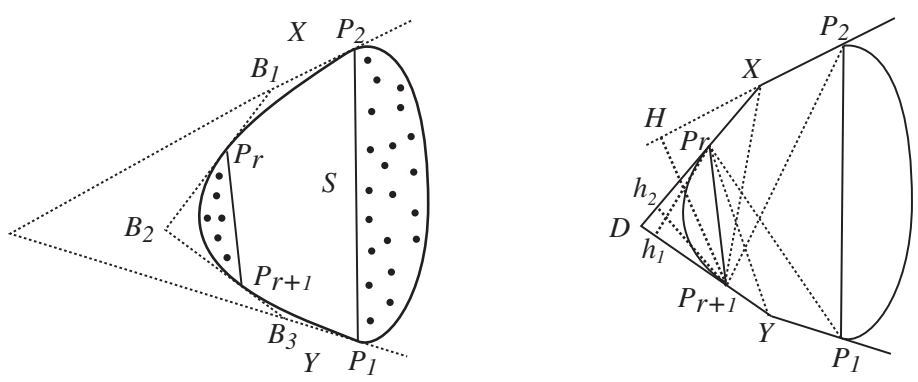

Fig. 8. The convex set $S$ and hex $\left(P_{2} X P_{r} P_{r+1} Y P_{1}\right)$. 
Put $\ell^{*}=\ell(\partial(S))$. By the symmetry of $h_{1}$ and $h_{2}$, we may assume that $h_{1} \leq h_{2}$. Then we obtain

$$
\begin{aligned}
\left(1-\alpha_{1}-\alpha_{r}\right) \operatorname{area}(S) \geq & \operatorname{area}\left(\operatorname{hex}\left(P_{2} X P_{r} P_{r+1} Y P_{1}\right)\right), \\
> & \operatorname{area}\left(\triangle P_{2} X P_{r+1}\right)+\operatorname{area}\left(\triangle X P_{r} P_{r+1}\right) \\
& +\operatorname{area}\left(\triangle P_{1} Y P_{r}\right)+\operatorname{area}\left(\triangle Y P_{r+1} P_{r}\right) \\
\geq & \frac{1}{2}\left|P_{2} X\right| h_{2}+\frac{1}{2}\left|X P_{r}\right| h_{2} \\
& +\frac{1}{2}\left|P_{1} Y\right| h_{1}+\frac{1}{2}\left|Y P_{r+1}\right| h_{1} \quad\left(\operatorname{by}\left|P_{r+1} H\right| \geq h_{2}\right) \\
= & \frac{1}{2} \ell\left(\operatorname{arc}\left(P_{2} P_{r}\right)\right) h_{2}+\frac{1}{2} \ell\left(\operatorname{arc}\left(P_{r+1} P_{1}\right)\right) h_{1} \\
\geq & \frac{1}{2} h_{1}\left(1-\alpha_{1}-\alpha_{r}\right) \ell^{*} .
\end{aligned}
$$

Therefore $\operatorname{area}(S)>\frac{1}{2} h_{1} \ell^{*}$. Then it follows from Lemma 5 that

$$
\text { area }\left(\text { lune }\left(P_{r} P_{r+1}\right)\right)<\frac{1}{2} h_{1} \ell\left(\operatorname{arc}\left(P_{r} P_{r+1}\right)\right)=\frac{1}{2} h_{1} \alpha_{r} \ell^{*}<\alpha_{r} \operatorname{area}(S) .
$$

This contradicts the assumption that area(lune $\left.\left(P_{r} P_{r+1}\right)\right) \geq \alpha_{r}$ area $(S)$.

We next consider the case of Fig. 9, where $K P_{2}, K P_{r}, B P_{1}$ and $B P_{r+1}$ are tangent to $S$ at $P_{2}, P_{r}, P_{1}$ and $P_{r+1}$, respectively. By considering lune $\left(P_{2} P_{r}\right)$ and $\triangle P_{2} K P_{r}$, we take a point $R$ on $P_{2} K \cup K P_{r}$ which satisfies the conditions of Lemma 5. Let $D$ be the intersection of the line passing through $B R$ and $\partial(S)$. We draw a line $A C$ tangent to $S$ at $D$, and take two points $X$ and $Y$ on $D A \cup A P_{1}$ and $D C \cup C P_{r+1}$, respectively, which satisfy the conditions of Lemma 5 (Fig. 9).

Let $E$ be the intersection of $A B$ and a line containing $R P_{2}$, and let $F$ be the intersection of $B C$ and a line containing $R P_{r}$. Let $k_{1}$ and $k_{2}$ denote the heights of $\triangle P_{1} E P_{2}$ and $\triangle P_{r} F P_{r+1}$, respectively. Then we obtain the following inequalities, where we may assume that two lines passing through $P_{1} P_{r+1}$ and $R P_{2}$ intersect at some point above $B D$ since otherwise two lines passing through $P_{1} P_{r+1}$ and $R P_{r}$ intersect at some point below $B D$ and the similar arguments given below can be applied:

$$
\begin{aligned}
& \ell\left(\operatorname{arc}\left(P_{1} P_{2}\right)\right)=\alpha_{1} \ell(\partial(S)), \quad \quad \text { area }\left(\text { lune }\left(P_{1} P_{2}\right)\right) \geq \alpha_{1} \text { area }(S), \\
& \ell\left(\operatorname{arc}\left(P_{r} P_{r+1}\right)\right)=\alpha_{r} \ell(\partial(S)), \quad \text { area }\left(\text { lune }\left(P_{r} P_{r+1}\right)\right) \geq \alpha_{r} \text { area }(S),
\end{aligned}
$$


Fig. 9. The convex set $S$ and octagon $\left(X P_{1} P_{2} R P_{r} P_{r+1} Y D\right)$. 


$$
\begin{aligned}
& \left.\ell\left(\operatorname{arc}\left(P_{2} P_{r}\right)\right)=\left|P_{2} R\right|+\left|R P_{r}\right|, \quad \text { area(lune }\left(P_{2} P_{r}\right)\right) \geq \operatorname{area}\left(\triangle P_{2} R P_{r}\right), \\
& \ell\left(\operatorname{arc}\left(D P_{1}\right)\right)=|D X|+\left|X P_{1}\right|, \quad \text { area }\left(\text { lune }\left(D P_{1}\right)\right) \geq \operatorname{area}\left(\triangle D X P_{1}\right), \\
& \ell\left(\operatorname{arc}\left(P_{r+1} D\right)\right)=\left|P_{r+1} Y\right|+|Y D|, \quad \text { area }\left(\text { lune }\left(P_{r+1} D\right)\right) \geq \operatorname{area}\left(\triangle P_{r+1} Y D\right), \\
& \operatorname{area}\left(\triangle X P_{1} P_{2}\right) \geq \frac{1}{2}\left|X P_{1}\right| k_{1}, \quad \operatorname{area}\left(\triangle X P_{2} R\right)=\frac{1}{2}\left|P_{2} R\right||X N|>\frac{1}{2}\left|P_{2} R\right| k_{1}, \\
& |R L| \geq|R Q| \geq\left|M P_{1}\right|>k_{1}, \quad \operatorname{area}(\triangle X R D)>\frac{1}{2}|X D| k_{1}, \\
& \left|P_{r} I\right|>\left|P_{r} G\right|>k_{2}, \quad \operatorname{area}\left(\triangle Y P_{r} P_{r+1}\right) \geq \frac{1}{2}\left|Y P_{r+1}\right| k_{2}, \\
& \operatorname{area}\left(\triangle Y R P_{r}\right)>\frac{1}{2}\left|R P_{r}\right| k_{2} \\
& |R H| \geq|R Q| \geq\left|M P_{1}\right|>k_{1}, \quad \operatorname{area}(\triangle Y R D)>\frac{1}{2}|Y D| k_{1} .
\end{aligned}
$$

Hence we have

$$
\begin{aligned}
& \operatorname{area}\left(\operatorname{octagon}\left(X P_{1} P_{2} R P_{r} P_{r+1} Y D\right)\right) \\
& \quad>\frac{1}{2}\left(|D X|+\left|X P_{1}\right|+\left|P_{2} R\right|+|Y D|\right) k_{1}+\frac{1}{2}\left(\left|R P_{r}\right|+\left|P_{r+1} Y\right|\right) k_{2} .
\end{aligned}
$$

We first assume that $k_{1} \leq k_{2}$. Put $\ell^{*}=\ell(\partial(S))$. Then

$$
\begin{aligned}
\left(1-\alpha_{1}\right. & \left.-\alpha_{r}\right) \operatorname{area}(S) \geq \operatorname{area}\left(\operatorname{octagon}\left(X P_{1} P_{2} R P_{r} P_{r+1} Y D\right)\right) \\
& >\frac{1}{2}\left(|D X|+\left|X P_{1}\right|+\left|P_{2} R\right|+|Y D|+\left|R P_{r}\right|+\left|P_{r+1} Y\right|\right) k_{1} \\
& =\left(1-\alpha_{1}-\alpha_{r}\right) \ell^{*} k_{1} .
\end{aligned}
$$

Therefore $\operatorname{area}(S) \geq \frac{1}{2} k_{1} \ell^{*}$, and thus

$$
\frac{\alpha_{1} \ell^{*} k_{1}}{2} \leq \alpha_{1} \operatorname{area}(S) \leq \operatorname{area}\left(\operatorname{lune}\left(P_{1} P_{2}\right)\right)<\frac{1}{2} \ell\left(\operatorname{arc}\left(P_{1} P_{2}\right)\right) k_{1}=\frac{\alpha_{1} \ell^{*} k_{1}}{2} .
$$

This is a contradiction.

We next assume $k_{2}<k_{1}$. Then we can similarly show that $\operatorname{area}(S) \geq \frac{1}{2} k_{2} \ell^{*}$. Thus we can derive a contradiction as follows:

$$
\frac{\alpha_{r} \ell^{*} k_{2}}{2} \leq \alpha_{r} \operatorname{area}(S) \leq \operatorname{area}\left(\operatorname{lune}\left(P_{r} P_{r+1}\right)\right)<\frac{1}{2} \ell\left(\operatorname{arc}\left(P_{r} P_{r+1}\right)\right) k_{2}=\frac{\alpha_{r} \ell^{*} k_{2}}{2} .
$$

Consequently the proof of the case of Fig. 9 is complete.

We next consider the case that $r=2$. In this case we have two configurations given in Figs. 10 and 11. Since these figures are very similar to Figs. 8 and 9, we can similarly derive a contradiction in each case by almost the same arguments given above. Consequently the lemma is proved.

Lemma 8. Let $S, n$ and $\alpha_{1}, \alpha_{2}, \ldots, \alpha_{n}$ be the same as in Theorem 2. Then there exist $n$ points $P_{1}, P_{2}, \ldots, P_{n}$ on $\partial(S)$ such that $\ell\left(\operatorname{arc}\left(P_{i} P_{i+1}\right)\right)=\alpha_{i} \ell(\partial(S))$ and area(lune $\left(P_{i} \times\right.$ $\left.\left.P_{i+1}\right)\right) \leq \alpha_{i}$ area $(S)$ for every $1 \leq i \leq n$, where $P_{n+1}=P_{1}$. 



Fig. 10. The convex set $S$ and hex $\left(D X P_{1} P_{2} P_{3} Y\right)$.

Proof. By Lemma 7 and by a new labeling of $\left\{P_{i}\right\}$, we may assume that there exist $n$ points $Q_{1}, \ldots, Q_{n}$ on $\partial(S)$ such that $\ell\left(\operatorname{arc}\left(Q_{i} Q_{i+1}\right)\right)=\alpha_{i} \ell(\partial(S))$ for all $1 \leq i \leq n$, area(lune $\left.\left(Q_{1} Q_{2}\right)\right)>\alpha_{1}$ area $(S)$ and area $\left(\right.$ lune $\left.\left(Q_{j} Q_{j+1}\right)\right) \leq \alpha_{j}$ area $(S)$ for all $2 \leq j \leq n$.

If there exist $n$ points $R_{1}, R_{2}, \ldots, R_{n}$ such that $\ell\left(\operatorname{arc}\left(R_{i} R_{i+1}\right)\right)=\alpha_{i} \ell(\partial(S))$ for all $1 \leq i \leq n$ and area(lune $\left.\left(R_{1} R_{2}\right)\right) \leq \alpha_{1}$ area $(S)$, then by Lemma 7 , when we continuously move $\left\{Q_{i}\right\}$ to $\left\{R_{i}\right\}$, we obtain the desired $n$ points $\left\{P_{i}\right\}$ satisfying the conditions of the lemma. So it is sufficient to show the existence such $n$ points $R_{1}, R_{2}, \ldots, R_{n}$. Moreover, if there exists two points $Y_{1}$ and $Y_{2}$ on $\partial(S)$ for which $\ell\left(\operatorname{arc}\left(Y_{1} Y_{2}\right)\right)=\alpha_{1} \ell(\partial(S))$ and area(lune $\left.\left(Y_{1} Y_{2}\right)\right) \leq \alpha_{1}$ area $(S)$, then add the remaining $n-2$ points $Y_{3}, \ldots, Y_{n}$ on $\partial(S)-\operatorname{arc}\left(Y_{1} Y_{2}\right)$ so that $\ell\left(\operatorname{arc}\left(Y_{i} Y_{i+1}\right)\right)=\alpha_{i} \ell(\partial(S))$ for $2 \leq i \leq n$. Then by Lemma 7 , these $n$ points $Y_{1}, Y_{2}, \ldots, Y_{n}$ are the desired $n$ points $\left\{R_{i}\right\}$.

We now show the existence of two such points $Y_{1}$ and $Y_{2}$. Since $\alpha_{1}<\frac{1}{2}$, there exist at least three points $Z_{1}, Z_{2}, \ldots, Z_{m}(m \geq 3)$ such that $\ell\left(\operatorname{arc}\left(Z_{i} Z_{i+1}\right)\right)=\alpha_{1} \ell(\partial(S))$ for all $1 \leq i \leq m-1$ and $\ell\left(\operatorname{arc}\left(Z_{m} Z_{1}\right)\right)<\alpha_{1} \ell(\partial(S))$. By applying Lemma 7 to the points $Z_{1}, Z_{2}, \ldots, Z_{m}$, we can say that at least one of lune $\left(Z_{1} Z_{2}\right)$ and lune $\left(Z_{2} Z_{3}\right)$ has area less than $\alpha_{1}$ area $(S)$. Therefore the lemma is proved.

Proof of Theorem 2. By Lemma 8, there exist $n$ points $\left\{P_{i}\right\}$ on $\partial(S)$ such that $\ell\left(\operatorname{arc}\left(P_{i} \times\right.\right.$ $\left.\left.P_{i+1}\right)\right)=\alpha_{i} \ell(\partial(S))$ and area(lune $\left.\left(P_{i} P_{i+1}\right)\right) \leq \alpha_{i}$ area $(S)$ for all $1 \leq i \leq n$. Let $P^{*}$ be the polygon with vertex set $\left\{P_{1}, P_{2}, \ldots, P_{n}\right\}$, and let $\left\{e_{1}, e_{2}, \ldots, e_{m}\right\}$ be the set of edges


Fig. 11. The convex set $S$ and hex $\left(D X P_{1} P_{2} P_{3} Y\right)$. 
$e_{k}=P_{j} P_{j+1}$ such that area(lune $\left.\left(P_{j} P_{j+1}\right)\right)<\alpha_{j}$ area $(S)$ for some $1 \leq j \leq n$. Define positive real numbers $\beta_{1}, \beta_{2}, \ldots, \beta_{m}$ by

$$
\beta_{k}=\alpha_{j} \operatorname{area}(S)-\operatorname{area}\left(\text { lune }\left(P_{j} P_{j+1}\right)\right) .
$$

Then by Theorem 4, $P^{*}$ can be partitioned into $m$ convex subsets $Q_{1}, Q_{2}, \ldots, Q_{m}$ such that each $Q_{k}$ has area $\beta_{k}$ and contains $e_{j}$. Since $S$ is a convex set, it is clear that lune $\left(P_{j} P_{j+1}\right) \cup Q_{k}$ is a convex subset. It is also obvious that lune $\left(P_{j} P_{j+1}\right) \cup Q_{k}$ has area $\alpha_{j}$ area $(S)$ and one continuous part of $\partial(S)$ with length $\ell\left(\operatorname{arc}\left(P_{j} P_{j+1}\right)\right)=\alpha_{i} \ell(\partial(S))$. Consequently the theorem is proved.

\section{References}

1. J. Akiyama, G. Nakamura and J. Urrutia. Perfect divisions of a cake. Proceedings of the Tenth Canadian Conference on Computational Geometry, pp. 114-115 (1998).

2. J. Akiyma, A. Kaneko, M. Kano, G. Nakamura, E. Rivera-Campo, S. Tokunaga and J. Urrutia. Radical perfect partitions of convex sets in the plane. In Discrete and Computational Geometry (ed. by J. Akiyama, M. Kano, and M. Urabe), pp. 1-13. Lecture Notes in Computer Science, vol. 1763. Springer-Verlag, Berlin (2000).

3. I. Bárány and J. Matoušek. Simultaneous partitions of measures by $k$-fans. Discrete Comput. Geom. 25 (2001), 317-334.

4. S. Bespamyatnikh, D. Kirkpatrick and J. Snoeyink. Generalizing ham sandwich cuts to equitable subdivisions. Discrete Comput. Geom. 24 (2000), 605-622.

5. H. Ito, H. Uehara and M. Yokoyama. Two-dimentional ham-sandwich theorem for partitioning into three convex pieces. In Discrete and Computational Geometry (ed. by J. Akiyama, M. Kano and M. Urabe), pp. 129-157. Lecture Notes in Computer Science, vol. 1763. Springer-Verlag, Berlin (2000).

6. A. Kaneko and M. Kano. Balanced partitions of two sets of points in the plane. Comput. Geom. Theory Appl. 13 (1999), 253-261.

7. A. Kaneko and M. Kano. Straight-line embeddings of rooted forests and semi-balanced partitions. Preprint.

8. A. Kaneko and M. Kano. Generalized balanced partitions of two sets of points in the plane. In Discrete and Computational Geometry (ed. by J. Akiyama, M. Kano and M. Urabe), pp. 1766-1786. Lecture Notes in Computer Science, vol. 2098. Springer-Verlag, Berlin (2001).

9. T. Sakai. Balanced convex partitions of measures in $\mathbf{R}^{2}$. Graphs Combin., 18 (2002), 169-192.

Received February 26, 2001, and in revised form February 11, 2002. Online publication July 24, 2002. 